\title{
Philonsorbonne
}

$10 \mid 2016$

Année 2015-2016

\section{Inhérence, immanence, ipséité : le premier Heidegger, lecteur des scolastiques}

\section{Mathieu EYCHENIÉ}

\section{(2) OpenEdition}

12 Journals

Édition électronique

URL : https://journals.openedition.org/philonsorbonne/783

DOI : 10.4000/philonsorbonne.783

ISSN : 2270-7336

Éditeur

Publications de la Sorbonne

\section{Édition imprimée}

Date de publication : 1 janvier 2016

Pagination : 49-68

ISSN : 1255-183X

\section{Référence électronique}

Mathieu EYCHENIÉ, «Inhérence, immanence, ipséité : le premier Heidegger, lecteur des scolastiques », Philonsorbonne [En ligne], 10 | 2016, mis en ligne le 19 janvier 2016, consulté le 08 juin 2021. URL : http://journals.openedition.org/philonsorbonne/783; DOI : https://doi.org/10.4000/philonsorbonne. 783

(c) Tous droits réservés 


\title{
Inhérence, immanence, ipséité : le premier Heidegger, lecteur des scolastiques
}

\author{
Mathieu EYCHENIÉ
}

\section{Introduction}

Nous prenons pour thème, dans cet article, la question de l'intériorité dans l'œuvre du premier Heidegger. Nous nous concentrerons donc sur la période 1916-1927: de la publication de la thèse d'habilitation de Heidegger, le Traité des catégories et de la signification chez Duns Scot, en 1916, à celle d'Être et temps en 1927. Nous nous intéresserons plus particulièrement à trois textes : la thèse d'habilitation, le cours de 1923-1924 intitulé Introduction à la recherche phénoménologique, et enfin les paragraphes d'Être et temps portant sur l'ipséité (paragraphe 64 : « Souci et ipséité ») et l'être-pour-la-mort (en particulier le paragraphe 48 : «Excédent, fin et totalité »).

L'enjeu de notre travail est de recomposer les différentes étapes de ce que l'on peut appeler la "destruction du sujet intérieur» opérée par Heidegger dans les années 1920, et de comprendre comment cette destruction (au sens heideggérien d'appropriation) rend possible la pensée du Dasein. La période étudiée (1916-1927) est particulièrement intéressante pour deux raisons. D'une part parce que la pensée heideggérienne du sujet évolue durant ces années (cette période est "coupée en deux » par ce que l'on appelle le premier tournant de la pensée heideggérienne, ou tournant herméneutique). D'autre part parce que cela nous donne l'occasion d'étudier la manière dont Heidegger lit les philosophes scolastiques. La confrontation avec les scolastiques, relativement à la question de l'intériorité $\mathrm{du}$ sujet, paraît a priori secondaire. Nous allons tenter de montrer, au contraire, qu'elle est absolument décisive. On verra ainsi que, durant la 
période 1916-1927, un scolastique se «cache » pour ainsi dire derrière chaque figure de l'intériorité que Heidegger réinvestit ou entend détruire : en 1916, Duns Scot derrière Husserl (au sens où Heidegger, dans sa thèse d'habilitation, lit Scot à partir de Husserl), en 1923-1924, Thomas d'Aquin derrière Descartes et Husserl.

Pour les besoins de notre propos, nous distinguerons deux figures de l'intériorité : l'inhérence et l'immanence. Nous tenterons de montrer que la pensée du Dasein, pleinement développée en 1927, ne peut s'élever que sur la base d'une destruction de ces deux conceptions de l'intériorité. Le Dasein de 1927 n'est ni un sujet d'inhérence ni un sujet immanent ; il est donc sans intériorité, du moins au sens traditionnel du terme. Nous essaierons en effet, pour terminer, d'identifier dans la pensée du Dasein de 1927 ce que l'on peut appeler des «reliquats» (ou «résidus») d'intériorité, qui rendent possible une nouvelle pensée de l'intériorité, adéquate à l'être du Dasein.

\section{Lecture de Duns Scot: la pensée immanente contre le sujet d'inhérence}

Dans la première partie de sa thèse d'habilitation, Heidegger commente différents textes de Duns Scot. Nous laissons de côté la question de savoir si Heidegger se montre fidèle à la pensée du philosophe scolastique ; il en ira de même par la suite, lorsque nous nous intéresserons à la lecture heideggérienne de Thomas d'Aquin. De toute évidence, dans sa thèse d'habilitation, Heidegger lit Duns Scot à partir de Husserl et d'une certaine philosophie néokantienne (Lotze, Rickert, Lask). L'héritage husserlien est particulièrement évident ; à la fin de l'introduction, Heidegger explique ainsi que :

« Ce dont il faut prendre conscience, c'est que la psychologie scolastique, précisément parce qu'elle n'est pas axée sur la réalité d'un psychisme caractérisé par son flux dynamique [das dynamisch-fließende Realpsychische], demeure initiée aux problèmes de principes d'une façon objectivo-noématique [in den prinzipiellen Problemen gegenständlich-noëmatisch]: situation qui favorise largement l'orientation du regard sur les phénomènes de l'intentionnalité [die Phänomene der Intentionalität] $»^{1}$.

Et si Heidegger a choisi d'étudier Duns Scot en particulier, c'est parce qu'il se montre extrêmement attentif, selon lui, à la "vie réelle » (realen Leben) :

1. Traité des catégories et de la signification chez Duns Scot, traduction par F. Gaboriau, Paris, Gallimard, 1970, p. 35. Pour le texte original, cf. Ga. 1, p. 205. Tout au long de l'article, le sigle $G a$. suivi d'un numéro renvoie aux différents tomes de l'édition complète (Gesamtausgabe) des œuvres de Heidegger en cours de parution chez Vittorio Klostermann à Francfort-sur-le-Main. 
«Plus que tous les Scolastiques avant lui il a trouvé une proximité (haecceitas) vaste et affinée pour ce qui est la vie réelle, pour sa multiplicité et sa possibilité de tension [seiner Mannigfaltigkeit und Spannungsmöglichkeit]. Ce qui ne l'empêche pas, en même temps, et avec la même facilité, de quitter cette plénitude de la vie [der Fülle des Lebens] pour se consacrer au monde abstrait des mathématiques. Les "figures que prend la vie" [Gestalten des Lebens] lui sont familières (dans la mesure où c'est possible au Moyen Âge) autant que le "gris-gris" de la philosophie $»^{2}$.

Heidegger expose la conception scotiste de l'intériorité dans le deuxième chapitre de la première partie de sa thèse, intitulé « Le verum et la réalité logique ». L'enjeu de la thèse est de délimiter les différents secteurs du « tout du pensable » (das All des Denkbaren), autrement dit de proposer une nouvelle table des catégories, post-aristotélicienne, afin, dans un second temps, de déterminer le "lieu logique » (logischen Ort) des significations. Heidegger, lisant Scot, pose donc exactement la question qui nous intéresse : celle du lieu des pensées (plus précisément des significations), donc la question de l'intériorité du sujet (qui est ici compris comme sujet pensant, et même, plus précisément, connaissant; une restriction qui évidemment n'aura plus cours dans les années 1920). Scot distingue différents secteurs d'effectivité (Wirklichkeit) : le domaine des êtres réels (realen), sensibles ou suprasensibles, et les domaines mathématique et logique, dont les objets ne sont pas suprasensibles mais non sensibles, et partant ont une certaine stabilité, une certaine persistance (Bestand) mais aucune existence réelle (reale Existenz). Dans le chapitre 2, Heidegger insiste sur ce qui différencie les choses réelles des objets logiques. L'objet logique, dit-il, est un ens logicum, ou encore un ens diminutum. Il pose clairement la question du lieu des êtres logiques :

«La réalité des choses [die reale Wirklichkeit] est celle qui ne dépend pas de l'âme (cujus esse non dependet ab anima). L'ens rationis est donc un ens in anima. La réalité logique appartient à l'"âme" [Die logische Wirklichkeit gehört der "Seele" an]. Comment concevoir de façon plus précise cette appartenance ? [...] L'expression ens in anima s'appliquant à la réalité logique ne désigne pas la réalité psychique [die psychische Realität]. La formule veut dire seulement ce qu'on exprime aujourd'hui en expliquant que le "sens noématique" [der "noematische Sinn"], l'intentionnalité du corrélat de conscience [die Intentionalität als Bewußtseinskorrelat] est inséparable [unabtrennbar] de la conscience sans pour autant s'y trouver réellement contenu [nicht reel in ihm enthalten $]^{3} »^{4}$.

\section{Ibid., p. 33 ; Ga. 1, p. 203.}

3. Heidegger fait ici référence à Husserl. Cf. notamment le paragraphe 97 des Idées directrices pour une phénoménologie et une philosophie phénoménologique pures, t. 1 , intitulé : «Que les moments hylétiques et noétiques sont des moments réels [reelle] du vécu, et les moments noématiques non-réels » (Idées directrices pour une phénoménologie et une philosophie phénoménologique pures, t. 1, traduction par P. Ricœur, Paris, Gallimard, 1950, p. 335). Dans le deuxième chapitre du Traité des catégories et de la signification chez Duns Scot, Heidegger ne distingue pas explicitement les termes real et reell. 
C'est cette dernière phrase qui est importante; et c'est le mot « réellement» qui est essentiel dans la critique de Scot, non l'idée de « contenu ».

On rencontre dans ce chapitre les deux figures de l'intériorité qui nous intéressent. Au sujet conçu comme contenant réellement ses pensées (la figure de l'intériorité à laquelle s'oppose Scot), nous donnons le nom de sujet d'inhérence. Par cette expression, nous renvoyons à la conception aristotélicienne de l'intériorité, c'est-à-dire à l'idée d'une inclusion réelle (ou: information, au sens large) des accidents (particuliers) dans le sujet, conçu comme hupokeimenon-subjectum ${ }^{5}$. Aristote, au chapitre II des Catégories, exprime cette idée d'inclusion réelle par la formule "être dans un sujet » (en hupokeimenôi einai $)^{6}$. À la thèse d'un sujet d'inhérence des pensées (l'idée selon laquelle les pensées seraient réellement incluses dans l'âme, conçue comme hupokeimenon-subjectum), Duns Scot oppose, selon Heidegger, une autre figure de l'intériorité : la pensée immanente. En effet, «[1]a théorie du reflet [Abbildtheorie] se heurte [...] à une difficulté insurmontable. En conséquence, Duns Scot la rejette et se décide en faveur d'une pensée immanente [für den Immanenzgedanken] ${ }^{7}$. Heidegger plaque ici sur le texte scotiste une thèse qui lui est étrangère : la thèse husserlienne des contenus de conscience, à laquelle la notion d'immanence, présente chez Husserl, renvoie en premier lieu ${ }^{8}$. Contrairement à ce qu'une lecture trop rapide pourrait laisser penser, Husserl, dès les Recherches logiques, ne refuse absolument pas d'appliquer à la pensée la notion de " contenu » (ce qui donne sens à l'idée d'une "pensée immanente »). Jocelyn Benoist l'explique dans son ouvrage intitulé Entre acte et sens. Recherches sur la théorie phénoménologique de la signification (2002). Dans l'introduction, il s'intéresse ainsi à ce que Husserl doit à un autre élève de Brentano, Twardowski. Husserl a critiqué l'opuscule de Twardowski, Zur Lehre vom Inhalt und Gegenstand der Vorstellungen, publié en 1894, mais, note Jocelyn Benoist, « cela dissimule bien mal tout ce qu'il lui emprunte $»^{9}$. Or,

4. Traité des catégories et de la signification chez Duns Scot, p. 103-105 ; Ga. 1, p. 275-277.

5. Nous laissons de côté, cependant, la question de savoir si Aristote a appliqué le modèle de l'inhérence à la pensée, à la connaissance.

6. Cf. sur ce point la distinction en hupokeimenôi einai - kath'hupokeimenou legetai introduite dans le chapitre II des Catégories.

7. Traité des catégories et de la signification chez Duns Scot, p. 101 ; Ga. 1, p. 273.

8. Pour les idées de «région-conscience» et d'immanence mutuelle de certains vécus, également impliquées dans la conception husserlienne du sujet immanent, cf. les deuxième et troisième moments de l'article. Par ailleurs, pour être absolument complet, il faudrait retracer l'origine (c'est-à-dire faire l'archéologie, au sens d'Alain de Libera) de la notion husserlienne d' " immanence ». Cela nécessiterait, entre autres choses, une confrontation avec Thomas d'Aquin, et sa conception de l'intellection comme «action immanente » de l'homme. Par la notion d' «immanence », nous nous référons cependant uniquement, dans ce travail, à la conception husserlienne de la pensée immanente, que Heidegger réinvestit dans sa thèse d'habilitation, avant de la déconstruire au début des années 1920.

9. J. Benoist, Entre acte et sens, Recherches sur la théorie phénoménologique de la signification, Paris, Vrin, 2002, p. 19. 
ce qu'il lui emprunte, c'est précisément la distinction entre «contenu» et «objet»: «ce que Husserl retient de Twardowski, c'est la structure maîtresse de sa conception de l'intentionalité significative - et en fait, comme le montrera la Recherche $\mathrm{V}$, de l'intentionalité en général - à savoir quelque chose comme la distinction entre le contenu et l'objet - mais sur le terrain purement idéal ${ }^{10}$. Pour Twardowski comme pour Husserl, le contenu fait office de médiation entre l'acte de représentation et l'objet. Husserl rompt cependant avec Twardowski relativement à la question de l'être de ce contenu: il le comprend comme une signification purement idéale, tandis que Twardowski, selon lui, le comprend encore comme une réalité psychique, inhérente à la conscience. Nous retrouvons ainsi, dans l'analyse de Jocelyn Benoist, la distinction que nous avons posée entre le sujet d'inhérence et la pensée immanente (Jocelyn Benoist préfère parler, quant à lui, des « deux sens de l'immanence ») :

« Twardowski n'est toutefois pas entièrement clair sur le statut de cette notion de "contenu". S'il prend bien soin de la distinguer de la représentation elle-même en tant qu'effectivité psychique, et paraît l'entendre en un sens quasi-sémantique, il semble tout de même la rattacher en quelque mesure à cette effectivité, dans le souci de lui donner un "site" ontologique. Par là même, il s'expose à la critique peu charitable de Husserl, qui l'accuse de confondre deux sens de l'immanence : celle, purement idéale, du "sens" à l'acte, et celle d'une inclusion réelle, effective, qui est celle du contenu psychique dans la conscience $»^{11}$.

Revenons à la thèse d'habilitation. Heidegger y distingue clairement l'inhérence et l'immanence. On ne peut pas comprendre l'évolution de sa conception de l'intériorité si l'on ne marque pas clairement la différence entre ces deux notions. Mais pour quelle raison Duns Scot en vient-il, d'après Heidegger, à rejeter l'idée d'un sujet d'inhérence des pensées ? Selon Scot, affirmer que les significations sont inhérentes au sujet implique d'adhérer à ce qu'il appelle les «théories du reflet», c'est-à-dire de concevoir le jugement vrai comme une «répétition », et non pas comme une " mise en forme » (Formung), de ce qui existe. Dire que les significations sont réellement contenues dans l'âme revient à dire qu'elles sont des choses réelles en elle-même; il faut donc concevoir ce qui existe dans l'âme comme une répétition, ou un reflet, de ce qui existe hors de l'âme. Duns Scot s'oppose ainsi aux théories du reflet parce qu'elles ne permettent pas de rendre compte de la possibilité des jugements vrais ${ }^{12}$ :

« Duns Scot soulève ici une difficulté devant laquelle se trouve finalement toute théorie du "reflet", lorsqu'elle travaille avec la connaissance comme avec

10. Ibid., p. 19-20.

11. Ibid., p. 19.

12. La question de la vérité est ainsi décisive. Ce point est capital, car on verra que c'est également pour rendre possible une pensée authentique de la vérité que Heidegger, au début des années 1920, détruit le sujet immanent. 
une représentation. Il n'est absolument pas possible en effet de comparer le sens du jugement avec les objets réels [den realen Objekten], car je ne sais rien des objets réels que, précisément, grâce à la connaissance, au jugement. Un objet qui n'est pas reconnu, n'est pas pour moi un objet. Nous n'allons pas audelà du contenu judicatoire [Urteilsgehalt] comme tel vers les objets réels euxmêmes $»^{13}$.

Les théories du reflet se heurtent à une contradiction : je ne peux pas savoir que le jugement «en moi » est vrai en le comparant avec les choses qui sont hors de moi, puisque, pour effectuer cette comparaison, il faudrait que je puisse me rapporter aux choses hors de moi indépendamment du jugement ; or cela est impossible, car le sujet connaissant ne se rapporte à des objets que par des jugements (seul le jugement peut, à proprement parler, être dit «vrai »). Ainsi, selon Scot, lu par Heidegger, parce que la position d'un sujet d'inhérence des significations implique de concevoir la représentation comme un "reflet» des objets existants, il faut rejeter le modèle de l'inhérence pour «sauver» la connaissance. Cela n'implique cependant pas de renoncer à l'intériorité, bien au contraire. Scot tient ensemble deux affirmations. D'une part, les significations ne sont pas des êtres psychologiques, elles appartiennent à un autre domaine d'êtres (le domaine logique). Mais d'autre part, ces significations sont bien intérieures au sujet connaissant, ce qui justifie que l'on parle de "pensée immanente » (Immanenzgedanken). D'une certaine manière, tout le geste scotiste est là : il tente de penser une intériorité non psychologique. Mieux, l'intériorité logique est la seule intériorité, l'intériorité psychologique n'est intérieure que de nom : elle conçoit en effet l'acte de juger comme une sortie hors des représentations psychologiques vers les objets réels, dont les représentations ne sont que des reflets. Elle situe donc les objets de pensée, les objets en tant qu'ils sont envisagés, jugés, connus, à l'extérieur de la pensée. La thèse de l'immanence, à l'inverse, affirme que le sens des objets, c'est-à-dire les objets en tant qu'ils sont envisagés, sont en moi. Duns Scot radicalise, en définitive, l'idée d'une intériorité de la pensée : il explique que les significations sont intérieures précisément parce qu'elles ne sont pas des choses réelles en moi. Ce faisant, il ne nie pas l'existence du monde extérieur : "La pensée immanente [Gedanke der Immanenz], correctement conçue, ne supprime pas la réalité et ne fait pas du monde extérieur un rêve $»^{14}$. Les objets, en tant qu'ils existent, sont hors de moi ; en revanche ils sont en moi en tant qu'ils sont envisagés, jugés, connus (leur signification est en moi) :

«Ens rationis signifie donc le contenu [den Gehalt], le sens des actes psychiques ; c'est un être [ein Sein] dans la conscience qui pense et considère, c'est l'ens cognitum, l'être pensé, l'être jugé. Il faut le discerner de ce qui est subjective in intellectu [...] Par ens in anima, il faut [...] comprendre le

13. Traité des catégories et de la signification chez Duns Scot, p. 101 ; Ga. 1, p. 272-273.

14. Ibid., p. 101 ; Ga. 1, p. 273. 
secundo consideratum, et donc pas la chose elle-même considérée dans la connaissance et la pensée du réel objectif [das Gegenständliche in objektiver Wirklichkeitserkenntnis und Gemeintheit] $»^{13}$.

\section{Retour à Thomas d'Aquin, par-delà Husserl et Descartes}

Ainsi, Duns Scot oppose au modèle de l'inhérence l'idée d'une pensée immanente. Analysons à présent les modalités de la destruction du sujet immanent opérée par Heidegger au début des années $1920^{16}$.

Lorsque Heidegger évoque l'idée d'un sujet immanent, il fait référence, nous l'avons dit, à Husserl. La destruction heideggérienne du sujet immanent prend (au moins) deux formes. D'une part, Heidegger refuse de considérer les significations comme des entités idéales, dont le sens d'être n'est pas véritablement élucidé ${ }^{17}$. Il explique ainsi, dans son cours de 1923 intitulé Ontologie, Herméneutique de la factivité, que

«[1]a significativité [Bedeutsamkeit] n'est pas une catégorie réale [Sachkategorie] qui regrouperait dans un domaine [Bereich] spécifique un certain nombre d'objets [Gegenstände] possédant, contrairement à d'autres, une teneur réale déterminée et qui délimiterait ce domaine en l'opposant à un autre. C'est un mode de l'être [ein Wie des Seins], et le catégorial de l'être-là (Dasein) du monde est à vrai dire centré sur elle $»^{18}$.

15. Ibid., p. 105 ; Ga. 1, p. 277-278.

16. Nous nous concentrerons essentiellement sur le cours de 1923-1924 intitulé Introduction à la recherche phénoménologique et consacrerons une remarque finale aux cours antérieurs.

17. Sur ce point, cf. notamment la notion d'« essence significative [bedeutungsmässigen] » au paragraphe 21 de la Recherche logique $V$ de Husserl.

18. Ontologie, Herméneutique de la factivité, traduction par A. Boutot, Paris, Gallimard, 2012, p. 116-117; $G a .63$, p. 86. Cf. également le même cours, p. 129 : «L'être-là du monde sera fixé terminologiquement par la significativité. Significatif veut dire : être, être-là sur le mode d'un signifier qui fait encontre de manière déterminée. Le terme ne désigne pas un étant qui serait là et signifierait en outre encore autre chose ; mais au contraire signifier en faisant encontre de manière déterminée, se tenir dans le signifier, c'est cela qui constitue l'être [macht das Sein aus]. Ce qu'il faut donc faire entendre, c'est que et comment la significativité constitue le Dasein mondain »; Ga. 63, p. 96-97. Notons que Heidegger reprendra ces éléments tels quels dans Être et temps. Cf. Etre et temps, traduction par E. Martineau, Édition numérique hors commerce, 1985, p. 132: «Lorsque de l'étant intramondain est découvert avec l'être du Dasein, autrement dit lorsqu'il est venu à compréhension, nous disons qu'il a du sens. Cependant, ce qui est compris, ce n'est pas en toute rigueur le sens, mais l'étant - ou l'être. Le sens est ce en quoi la compréhensibilité de quelque chose se tient. (...) Le sens est un existential du Dasein, non pas une propriété qui s'attache à l'étant [eine Eigenschaft, die am Seienden haftet], est "derrière" lui ["hinter" ihm liegt] ou flotte quelque part comme "règne intermédiaire" [Zwischenreich]. De sens, le Dasein n'en "a", que pour autant que l'ouverture de l'être-au-monde [die Erschlossenheit des In-der-Welt-seins] est "remplissable" par l'étant découvrable en elle. Seul le Dasein, par suite, peut être sensé ou in-sensé»; Sein und Zeit, Tübingen, Max Niemeyer Verlag, 2006, p. 151. 
Pour Heidegger, la signification n'est une entité en aucun sens (elle n'est ni un étant sensible ou suprasensible, ni une valeur, au sens néokantien du terme). D'une certaine façon cependant, en disant cela, Heidegger ne critique pas Husserl mais le radicalise : il radicalise sa théorie de l'acte (contre son objectivisme sémantique) ${ }^{19}$.

Heidegger se livre par ailleurs à une seconde critique, peut-être plus radicale, de l'idée d'immanence : il s'oppose à la conception husserlienne de la conscience pure comme champ d'étude pour une science déterminée, autrement dit comme une certaine région qu'il est possible d'étudier scientifiquement (pour Husserl, la conscience pure est même l'Urregion ${ }^{20}$ ). Selon la lecture de Heidegger, la pensée, chez Husserl, est immanente en tant qu'elle prend «place» dans une «région réale», une Sachregion (ce que l'on peut «traduire » ainsi : une région d'étants-sous-la-main, Vorhandenen), la région des vécus (Erlebnisse), qui font l'objet d'une étude scientifique se donnant pour modèle de rigueur une science déterminée (les mathématiques).

Analysons en détail la destruction heideggérienne de la «régionconscience» husserlienne. Suivons pour ce faire l'Introduction à la recherche phénoménologique (cours du semestre d'hiver 1923-1924). Dans ce cours, Heidegger explique que la conception husserlienne de la conscience comme une région déterminée est fondée sur le privilège accordé, de manière injustifiée, au comportement théorique du Dasein. Pour comprendre Husserl cependant, il faut revenir à Descartes. Heidegger ne nie absolument pas qu'il existe des différences entre les conceptions cartésienne et husserlienne de la conscience ; ces derniers s'accordent néanmoins sur la réponse à donner à la question principale : celle de l'être de la conscience. Ainsi,

« [o]n aurait tort d'assimiler la doctrine de Husserl à celle de Descartes. Il faut souligner d'emblée en effet une différence fondamentale dans la manière dont Husserl appréhende la conscience quant à son contenu [wie Husserl inhaltlich das Bewußtsein $f a \beta t]$; en revanche, concernant le traitement de la question de l'être de la conscience et le sens de l'esse certum [hinsichtlich der Erledigung der Seinsfrage des Bewußtseins und im Hinblick auf den Sinn des esse certum], Husserl se meut entièrement dans la perspective ouverte par Descartes, et veut même, aujourd'hui plus que jamais, aller plus loin que Descartes pour saisir cette perspective de façon enfin absolue $»^{21}$.

19. Cf. sur ce point J. Benoist, « Heidegger, les sens du sens et l'illusion herméneutique », in Heidegger, M. Caron (dir.), Paris, Les Éditions du Cerf, 2006, p. 340 : « à plus d'un titre, (...) l'analyse heideggérienne du sens [dans Être et temps] peut être présentée comme une réactivation et aussi une radicalisation critique d'une face de l'analyse husserlienne - la face intentionaliste, que Heidegger joue en quelque sorte contre la face objectiviste sémantique et pour ainsi dire en correction d'elle, afin de surmonter ses apories ».

20. Cf. Husserl, Idées directrices pour une phénoménologie et une philosophie phénoménologique pures, t. 1, paragraphe 76.

21. Introduction à la recherche phénoménologique, traduction par A. Boutot, Paris, Gallimard, 2013, p. 276 ; Ga. 17, p. 254. 
En quoi consiste l'accord fondamental, principiel, de Descartes et Husserl ? L'un et l'autre posent la question de l'être de la conscience à partir d'une guise d'être particulière : le souci de certitude, qui est un mode de ce que Heidegger appelle le souci de la connaissance connue (Sorge um erkannte Erkenntnis), qui constitue lui-même un comportement théorique particulier. Le Dasein, dans cette guise d'être, est préoccupé par la connaissance théorique et par la préservation de cette connaissance. Selon Heidegger, c'est chez Descartes que cette préoccupation, ce souci, se manifeste pour la première fois en tant que tel : " La guise concrète de l'être du souci d'une connaissance connue [Die konkrete Weise des Seins der Sorge um erkannte Erkenntnis], dans laquelle la conscience s'ouvre comme champ [das Bewußtsein als Feld erschließt], nous est donnée au grand jour [ist uns offen gegeben] dans les recherches de Descartes $»^{22}$. Pour comprendre pleinement la manifestation de ce souci chez Descartes (et c'est ce point qui va nous intéresser tout particulièrement), il faut, toutefois, faire encore un pas en arrière, en amont de Descartes. Selon Heidegger, le souci de la connaissance connue n'a pu apparaître, chez Descartes, que sur la base de l'ontologie scolastique, et plus spécifiquement thomiste, qui considère l'âme connaissante comme un être créé, un esse creatum. Afin de comprendre la manifestation de ce souci (qui conditionne la conception de la conscience comme une région d'étants-sous-la-main), il est nécessaire, donc, de faire retour à Thomas d'Aquin. D'après Heidegger, ni la philosophie grecque, ni Thomas d'Aquin, n'ont fait de la conscience l'objet d'une recherche explicite (la question essentielle ici étant celle du rapport à soi ${ }^{23}$ ). Pour autant, la thématisation de la conscience chez Descartes et Husserl n'a été possible que sur la base de l'ontologie thomiste.

Comment Heidegger en vient-il à une telle conclusion? La question de la vérité est ici centrale. Le souci de la connaissance connue implique en effet, chez Descartes, une certaine conception de la vérité : la vérité est conçue comme être-jugé. Ceci constitue un mouvement de recul par rapport à l'alètheia grecque. La vérité est désormais "située » dans l'intellect: Descartes reprend cette idée à Thomas qui, dans le De veritate, affirme que la vérité est « ultimement» située dans l'intellect divin. Plus précisément, la vérité est conçue chez Descartes comme être-jugé « réglé » (la vérité fait en effet l'objet d'une régulation; au début de la "Méditation troisième », Descartes énonce une règle générale de vérité, qui définit le mode d'accès à la vérité). Selon Heidegger, cette conception de la vérité comme être-jugé réglé n'a cependant de sens chez Descartes que si la pensée est comprise comme un esse creatum, autrement dit uniquement dans le cadre de l'ontologie thomiste. En effet, d'une part, l'homme cherche à s'assurer de la vérité en posant une règle parce que l'accès à la vérité, comprise comme un bonum, permet à cet être créé d'atteindre une certaine forme de perfection, la

22. Ibid., p. 123 ; Ga. 17, p. 106.

23. Nous reviendrons sur la question du rapport à soi (un autre sens de l'immanence) dans le troisième et dernier moment de l'article. 
perfectio hominis ; d'autre part, la vérité doit être interprétée comme un êtrejugé, parce que l'être de l'homme est rattaché à la perfection suprême par le jugement (celui-ci constitue en effet un mode du vouloir, et la volonté me fait connaître que je porte l'image et la ressemblance de Dieu). Heidegger démontre ainsi que la vérité comprise comme être-jugé réglé (donc le souci de la connaissance connue, et partant la conception de la conscience comme une région d'étants sous-la-main) n'a pu se manifester que parce que l'intellect humain a été interprété par Thomas comme un esse creatum. Certes, Husserl, contrairement à Descartes, ne considère pas la conscience comme un esse creatum, mais comme un ens regionale, un être régional. Pour autant, cela ne signifie pas que Husserl rompt avec Thomas d'Aquin, mais au contraire qu'il recouvre cet héritage thomasien; ce recouvrement rend alors possible une radicalisation de la conception originelle. Ainsi,

«Husserl ne s'exprime pas [...] sur la base d'une claire perception des connexions historiques [einer klaren Einsicht in die geschichtlichen Zusammenhänge], mais en étant mû par le refus de parler de Dieu et de l'âme dès le début de la philosophie. Cela montre ce que veut la phénoménologie ellemême : poser la conscience en reprenant le cogito sum en tant que certum comme une chose allant de soi [die Ansetzung des Bewußtseins im Sinne der Aufnahme des cogito sum als certum in der Weise einer Selbstverständlichkeit], et cela en procédant à un élargissement [Erweiterung] fondamental qui a forme d'un déracinement [Entwurzelung] $»^{24}$.

L'élargissement (c'est-à-dire l'élargissement de la recherche scientifique au tout de la "région-conscience»; chez Descartes, en effet, la certitudo a simplement pour objet le cogito ainsi que certaines cogitationes isolées) est rendu possible par le déracinement (l'oubli de l'héritage thomasien).

Descartes rompt néanmoins avec Thomas sur un point fondamental : il transforme le verum en certum. Thomas considère seulement le vrai comme un être-jugé ; Descartes (et après lui Husserl) va en réalité plus loin en le considérant comme un être-certain. C'est dire que Descartes ne fait pas seulement de la conscience l'objet d'une recherche scientifique, mais qu'il érige en modèle de toutes les sciences l'arithmétique et la géométrie, qui appréhendent leurs objets selon une evidens et une clara perceptio. On retrouve ce geste chez Husserl, qui dans sa méthode phénoménologique accorde un rôle central à l'évidence, pensée comme recouvrement du visé. Chez Descartes donc, comme chez Husserl, l'être n'est appréhendé que par le détour du certum, ce qui revient selon Heidegger à le soumettre à la logique formelle. Ce geste est absolument « funeste» car il signifie ni plus ni moins que renoncer à interroger le sens d'être de la conscience; ce qui intéresse Descartes et Husserl, en définitive, ce n'est pas la chose même, mais l'établissement de propositions certaines. Ainsi, ce que Heidegger «reproche » à Descartes, ce n'est pas directement de faire de la pensée une

24. Introduction à la recherche phénoménologique, p. 290 ; Ga. 17, p. 268. 
" chose », mais au contraire de se désintéresser de cette chose, de ne pas poser la question de l'être de cette chose :

«Pour nous, la question du revirement [du verum en certum] signifie voir que ce dernier ne veut rien dire d'autre que la relégation de la conscience dans la sphère catégoriale de l'ontologie formelle [die Versetzung des Bewußtseins in die kategoriale Sphäre des formal Ontologischen]. [...] Descartes a donné à cette relégation une expression prégnante dans la mesure où lui-même ne prend pas le cogitare comme une chose [Sache] qui aurait été trouvée (une chose ayant la structure d'une cause dont on a à débattre); ce qui lui importe est d'avoir trouvé une proposition certaine [einen gewissen Satz]. Cette proposition du cogito est pour Descartes une proposition logique et formelle [eines formal-logischen Satzes] qui vaut de cette chose [Ding] que nous pouvons caractériser en bref comme chose pensante [Denkding] $»^{25}$.

De la même façon, Husserl ne pose pas la question de l'être de la conscience ; ce qui l'intéresse est de circonscrire une certaine région qui peut donner lieu à des propositions certaines. Ainsi,

«[1]a prédominance du souci de certitude [Die Vorherrschaft der Sorge der Gewißheit] se manifeste [chez Husserl] en ceci qu'on s'en tient, concernant le champ thématique "conscience" [bezüglich des thematischen Feldes "Bewußtsein"], à des déterminations de l'ontologie traditionnelle, et plus encore à celles de la logique formelle. [...] La conscience est la région des vécus [Bewußtsein ist Region der Erlebnisse]. La vie elle-même, en tant qu'intégralité des vécus [als die Gesamtheit der Erlebnisse], est déterminée comme région réale de ces faits particuliers [als Sachregion dieser einzelnen Fakten]. On ne parvient pas à entendre la vie elle-même dans son être véritable ni à répondre à la question de son caractère d'être $»^{26}$.

Pour Heidegger, le souci de certitude atteint son apogée, chez Husserl, dans la réduction éidétique, c'est-à-dire dans l'application de catégories telles que "genre, espèce, singularité éidétique, différence spécifique [Gattung, Art, eidetische Singularität, spezifische Differenz] ${ }^{27}$ à la conscience, donc de "catégories qui ont leur sol à elles [ihren bestimmten Boden haben] et qui ne disent rien d'un être tel que la conscience ${ }^{28}$.

Nous nous sommes concentrés essentiellement, dans ce second moment, sur l'Introduction à la recherche phénoménologique de 1923-1924. Faisons, pour terminer, une remarque relative aux cours antérieurs (mais postérieurs au tournant herméneutique). Sans trop entrer dans les détails ${ }^{29}$, signalons que

25. Ibid., p. 269-270; Ga. 17, p. 247-248.

26. Ibid., p. 295-296; Ga. 17, p. 273-275.

27. Ibid., p. 296 ; Ga. 17, p. 274.

28. Ibid., p. 296 ; Ga. 17, p. 274.

29. On pourrait, en effet, consacrer une étude entière à la "phénoménologie de la vie » caractéristique de la première période d'enseignement de Heidegger à Fribourg (1919-1923). 
la rupture avec Husserl, relativement à la question de l'immanence, est en un sens consommée des 1919. On lit en effet dans Les problèmes fondamentaux de la phénoménologie de 1919-1920 : «L'examen de la sphère totale de la vie pure n'est ni un examen extérieur ni un examen intérieur, ni transcendant ni immanent $»^{30}$. En 1919-1920, Heidegger refuse donc déjà d'appliquer à la sphère de la vie facticielle l'idée d' «intériorité" comprise comme «immanence». À cette même époque, il s'oppose également à toute présentation "figurée » de la conscience, c'est-à-dire à toute conception de la conscience comme un certain «espace» (une conception corrélée à la compréhension de la conscience comme une région d'étants sous-la-main). C'est l'un des motifs de la critique de la conception diltheyenne de la conscience dans le cours de l'été 1920, Phénoménologie de l'intuition et de l'expression. La critique de Heidegger porte, notamment, sur la notion de Zusammenhang. Dilthey caractérise par ce terme l'être de la vie psychique, en tant qu'unité immanente à ses parties. Dans le cours cité, Heidegger considère cette caractérisation comme " esthétique »; elle donne une image situationnelle de la vie, elle comprend le vécu comme un certain état, partant comme un certain espace, alors que sa réalité est essentiellement historique, temporelle ${ }^{31}$. En 1919-1920, certains éléments de la destruction du sujet immanent sont donc déjà présents.

Pour autant, la destruction ne semble pas totalement effectuée. Ainsi, dans le cours de 1919-1920 déjà cité, Heidegger emploie certaines formules qui peuvent prêter à confusion, telle que celle de «monde de soi » $(\text { Selbstwelt })^{32}$. Mieux, il parle de ce monde comme de 1'Urgebiet ${ }^{33}$, et, en 1923, du Dasein comme d'une certaine Region ou d'un certain Bezirk:

«L'herméneutique prend à chaque fois pour objet [Gegenstand] thématique le Dasein propre - qu'elle interroge dans son caractère d'être et ses structures phénoménales; donc, tout en ayant en vue une systématique régionale universelle [universale regionale Systematik], elle en extrait un secteur [Bezirk] déterminé pour conduire une recherche systématique déterminée. Pour fixer terminologiquement, délimiter et s'approprier cette région d'être [Seinsregion], on a évité, et on évitera dorénavant l'expression Dasein humain, être humain $»^{34}$.

Certes, on peut donner au terme de "région » un sens plus ou moins précis; parler du Dasein comme d'une région (au sens où il est un type

30. Grundprobleme der Phänomenologie (1919/20), Ga.58, p. 253 : Die Betrachtung der ganzen Sphäre des reinen Lebens ist weder Außen- noch Innenbetrachtung, weder transzendent noch immanent.

31. Cf. Phénoménologie de l'intuition et de l'expression, Théorie de la formation des concepts philosophiques, traduction par G. Fagniez, Paris, Gallimard, 2014, p. 194 ; Ga. 59, p. 167.

32. Notons qu'il renoncera à ce vocabulaire par la suite.

33. Cf. par exemple le titre du paragraphe 19 : Ein Grundproblem der Phänomenologie: die Zugänglichkeit des Ursprungsgebietes vom faktischen Leben aus (Ga. 58, p. 81).

34. Ontologie, Herméneutique de la factivité, p. 48 ; Ga. 63, p. 25-26. 
d'étant particulier, non une Sachregion) ne le ramène donc pas ipso facto dans le régime de la Vorhandenheit. On peut présumer, toutefois, que Heidegger, dans le courant des années 1920, tend à appliquer au Dasein un vocabulaire temporel et non plus spatial. En suivant Jean-François Marquet et son article "Genèse et développement d'un thème : l'isolement», on dira que la raison de cette "tendance» réside dans la prise en charge du problème de la mort, et de son corrélatif, l'instant, à partir des années 1922-23 : «L'isolement ${ }^{35}$ [du Dasein], de 1919 à 1929, n'a [...] pas cessé d'être au cœur de la pensée heideggérienne, mais il a cessé peu à peu de désigner une région de l'enquête phénoménologique pour évoquer un événement perpétuellement imminent, même dans l'esquive : celui de la décision où je serai(s) inconditionnellement moi-même $»^{36}$.

\section{Les « reliquats»de l'intériorité : réflexion, isolement et totalité (fermeture)}

Nous avons analysé les destructions successives du sujet d'inhérence et $\mathrm{du}$ sujet immanent (en nous concentrant, à chaque fois, sur le dialogue engagé avec la philosophie scolastique). Si le Dasein n'est ni un sujet d'inhérence ni une pensée immanente, il semblerait naturel de conclure, plus généralement, qu'il est sans intériorité. Le sens que Heidegger donne au terme Dasein semble en vérité exclure a priori toute idée d'intériorité : en tant qu'être-le-là, le Dasein est essentiellement au-monde, ce qui implique qu'il n'est pas une sphère intérieure « reliée » au «monde extérieur ». Ce point est établi dès 1919-1920 : « Notre vie est seulement comme vie dans la mesure où elle vit dans un monde $»^{37}$. Le Dasein est toujours-déjà au-dehors. Pour autant, n'est-ce pas conclure trop rapidement à l'absence d'intériorité du Dasein ? Ne pourrait-on pas penser un nouveau sens de l'intériorité, distinct du sens traditionnel, un sens de l'intériorité adéquat à l'être du Dasein en tant que Dasein ? Pour le dire en un mot: bien que cela semble a priori contradictoire, ne pourrait-on pas penser une intériorité de l'être-aumonde?

C'est précisément, selon nous, ce que tente de faire Heidegger. Dans les années 1920, il tente de donner un nouveau sens à l'idée d'intériorité, un sens conforme à l'être du Dasein conçu comme être-au-monde. On le comprend en se focalisant sur la fin (toute privisoire) du parcours : $\hat{E}$ tre et temps (ainsi que sur les cours de la même période). En conclusion de cet article, nous tenterons ainsi de «localiser», dans l'Hauptwerk de 1927, ce

35. Nous reviendrons sur ce thème dans le troisième moment de l'article.

36. J.-F. Marquet, « Genèse et développement d'un thème : l'isolement », in Heidegger 19191929, De l'herméneutique de la facticité à la métaphysique du Dasein, J.-F. Courtine (dir.), Paris, Vrin, 1996, p. 203-204.

37. Ga. 58, p. 34 : unser Leben ist nur als Leben, insofern es in einer Welt lebt. 
que nous appelons des « reliquats » ou « résidus » d'intériorité, qui sont mis au service d'une nouvelle pensée de l'intériorité. En quel sens le Dasein possède-t-il encore, en 1927, une forme d'intériorité ?

La réflexion constitue selon nous le premier «résidu»d'intériorité présent en 1927. Heidegger, nous l'avons dit, a dans les années précédant la publication d'Etre et temps détruit le sujet immanent. Il conserve pourtant, en 1927, quelque chose du sujet immanent ; ce qui constitue peut-être le sens principal de l'immanence, que nous n'avons pas évoqué jusqu'à présent : la propriété du Dasein d'être conjointement en possession de soi-même $(\text { Sich-mit-haben })^{38}$. Cette notion de Sich-mit-haben est à mettre en rapport avec celle de « souci » (Sorge) : le souci, qui constitue l'être du Dasein, est en effet toujours, pour Heidegger, souci de soi (y compris dans l'échéance, la Verfallenheit $)^{39}$. En 1927, Heidegger reste donc, de ce point de vue, cartésien et husserlien (et même thomasien, puisque pour Thomas l'intellection est en même temps intellection de soi). Cette conservation du sens principal de l'immanence apparaît clairement dans l'Introduction à la recherche phénoménologique de 1923-1924, notamment lorsque Heidegger commente la formule de Descartes cogito me videre:

«On voit [...] que Descartes comprend d'emblée le cogitare comme une guise d'être spécifique dans laquelle on est conjointement en possession de soi-même [ein eigentümliches Sein, das ist in der Weise des Seins im Wie des Sich-mit-habens], une guise dans laquelle celui qui est est du même coup primordialement en rapport à soi et se possède ainsi soi-même [sich mithat $]{ }^{40}$.

Husserl entrevoit le phénomène de l'être-avec-soi. Il conçoit celui-ci comme un acte de réflexion. Pour Husserl, l'immanence de la conscience se révèle dans l'acte de réflexion, compris comme une relation d'inclusion réelle du vécu réflechissant dans le vécu réfléchi, à l'intérieur de la « régionconscience ».

Heidegger rompt-il avec la conception husserlienne de la réflexion ? Oui, de toute évidence, car si Husserl, selon Heidegger, va plus loin que Descartes dans sa compréhension du «se posséder conjointement soimême » (il utilise en effet la réflexion comme un instrument destiné à configuer le cheminement de la phénoménologie, et il comprend cettedernière comme rapport à soi et au monde, en vertu de sa thématisation de l'intentionnalité), il méconnaît cependant le sens de la possession de soi, parce qu'il la comprend comme un rapport, une relation d'inclusion de deux étants à l'intérieur d'une région d'étants-sous-la-main, en laissant ininterrogé

\footnotetext{
38. Cette possession du Dasein par lui-même est également exprimée par les notions d'《 être-à-chaque-fois » (Jeweiligkeit) et de «mienneté » (Jemeinigkeit). Elle se manifeste ultimement dans le phénomène de la conscience (Gewissen), qui appelle le Dasein à être luimême (cf. Etre et temps, paragraphes 54-60).

39. Un tel retrait par rapport à soi n'étant possible que parce que l'être lui-même se donne comme retrait.

40. Introduction à la recherche phénoménologique, p. 271 ; Ga. 17, p. 249.
} 
le sens d'être de ces étants ${ }^{41}$. Dans sa compréhension du phénomène de la réflexion, Husserl accorde en réalité une nouvelle fois, de manière injustifiée, un privilège au comportement théorique : il la comprend comme un regard dirigé vers soi-même, autrement dit comme une perception de soi par soi. En 1927, Heidegger conserve l'idée de possession de soi, mais celle-ci est comprise comme Auslegung, c'est-à-dire comme explicitation ou interprétation du projet du Dasein par lui-même, et non pas comme la relation d'un étant à un autre au sein d'un ensemble d'étants-sous-lamain $^{42}$. La propriété du Dasein d'être à chaque fois lui-même est certes encore exprimée en terme de «possession» (ou plutôt d' "avoir») ; mais ce «s'avoir-avec-soi » (Sich-mit-haben) n'a pas le sens d'une mise à disposition, d'une objectivation, d'une saisie perceptive, il est notamment l'expression de la facticité du Dasein, donc de sa «passivité » (en tant que le Dasein ne constitue pas pour lui-même un fondement, au sens d'archè).

Heidegger, cependant, continue, en 1927, d'appliquer la notion de «réflexion» au Dasein; il donne toutefois à ce terme un sens nouveau : un sens optique. Il l'explique dans Les problèmes fondamentaux de la phénoménologie de 1927 :

«Le Soi [Selbst] est présent au Dasein lui-même sans réflexion [Reflexion] et sans perception interne [innere Wahrnehmung], antérieurement à toute réflexion. [...] On peut cependant nommer, avec pertinence, "réflexion", la manière dont le Soi se dévoile à soi-même dans le Dasein facticiel, pourvu seulement que l'on n'entende pas par là, comme c'est généralement le cas, l'auto-fascination de l'ego replié sur lui-même, mais un ensemble de relations $\left[\right.$ Zusammenhang $\left.{ }^{43}\right]$, comme l'atteste l'acception optique du terme. Se réfléchir

41. Sur ce point, cf. Prolégomènes à l'histoire du concept de temps (semestre d'été 1925), traduction par A. Boutot, Paris, Gallimard, 2006, p. 156 : «Immanence [chez Husserl] veut dire formellement d'abord: être dans un autre [in einem anderen sein]. Cette propriété d'immanence est attribuée à la région conscience, à la région du vécu [der Bewußtseins-, Erlebnisregion], et cela en considérant les actes de saisie, les actes réflexifs qui s'orientent sur d'autres actes, sur d'autres vécus. (...) Il y a, entre le vécu réfléchissant et le réfléchi [Zwischen dem reflektierenden Erlebnis und dem Reflektierten, dem in der Reflexion Gegenständlichen], une relation d'inclusion réelle [eine Beziehung des reellen ineinander Beschlossenseins]. (...) L'immanence n'est donc pas une détermination de l'étant en luimême quant à son être [keine Bestimmung des Seienden an ihm selbst hinsichtlich seines Seins], mais c'est une relation entre deux étants à l'intérieur de la région vécu ou conscience [innerhalb der Region Erlebnis oder Bewußtsein]. Cette relation est caractérisée comme inclusion réelle [reelles Ineinander], mais justement rien n'est dit sur l'être de cet être-inclus, sur la réellité [Reellität], sur l'étant en totalité de cette région [das Seiende im Ganzen dieser Region]. C'est un rapport d'être entre étants [ein Seinsverhältnis von Seiendem], mais non pas l'être comme tel qui est ici déterminé »; Ga. 20, p. 142.

42. Heidegger substitue d'une certaine manière au cercle de la réflexion husserlienne un autre cercle : le cercle herméneutique. Il se montre cependant prudent avec la notion de " cercle », précisément à cause de son sens spatial. Il écrit ainsi au paragraphe 32 d'Être et temps que " si l'on songe que le "cercle" appartient ontologiquement à un mode d'être de l'être-sous-lamain [Vorhandenheit] (à la réalité subsistante [Bestand]), on devra en général éviter de caractériser ontologiquement par un tel phénomène quelque chose comme le Dasein» (p. 134); Sein und Zeit, p. 153.

43. Notons que l'on retrouve ici le terme employé par Dilthey. 
signifie alors se réfracter sur quelque chose, en rejaillir, c'est-à-dire se montrer en se reflétant sur quelque chose $»^{44}$.

En 1927, se réfléchir ne signifie plus se percevoir soi-même en se «retournant » vers une région déterminée, mais se découvrir, se dévoiler soimême dans le monde, comme être-au-monde ${ }^{45}$.

La réflexion constitue ainsi le premier « reliquat» d'intériorité. On peut identifier un second "résidu»: l'isolement. Celui-ci apparaît notamment au paragraphe 64 d'Être et temps, intitulé «Souci et ipséité » [Sorge und Selbstheit]. Heidegger distingue en réalité, dans ce paragraphe, deux formes d'isolement du Dasein. La première forme rejoint la notion traditionnelle d'intériorité, autrement dit l'idée d'une "région-conscience». Heidegger s'intéresse à la conception kantienne du sujet, et il affirme que si Kant a reconnu que le «Je pense » est toujours un «Je pense quelque chose », il n'a pas interrogé le sens d'être de cet «accompagnement» de la pensée par le «quelque chose»; il manque ainsi le phénomène de l'être-au-monde, et continue de distinguer la « région-sujet », le sujet intérieur, isolé (isoliert), du monde (dont il manque également, par conséquent, le sens d'être). Ainsi, chez Kant,

« [1] dire-Je vise l'étant que je suis à chaque fois en tant que "Je-suisdans-un-monde". Mais Kant n'aperçut pas le phénomène du monde, et c'est pourquoi il fut parfaitement conséquent en tenant les "représentations" à distance de la teneur "apriorique" du "Je pense". Seulement, le Je s'en trouva de nouveau réduit à un sujet isolé [isoliert], accompagnant les représentations selon une guise tout à fait indéterminée ontologiquement ${ }^{46}$.

Le Dasein, dit Heidegger, n'est pas isolé en ce sens ; il ne constitue pas une région, une intériorité isolée, un espace au sein duquel on pourrait distinguer des pensées, des vécus, etc. En 1927, le rapport est en réalité inversé : rien n'est dans le Dasein, c'est celui-ci désormais qui est dit in : inder-Welt-sein. Le in, ici, n'a cependant pas le sens d'une inclusion spatiale, d'un «être-contenu-dans", il signifie "habiter», "séjourner auprès de». Heidegger l'explique au pagraphe 12 d'Etre et temps :

« nous inclinons à comprendre cet être-à... [In-Sein] comme un être dans... [Sein in]. Ce dernier terme nomme le mode d'être d'un étant qui est dans un autre comme l'eau dans le verre, le vêtement dans l'armoire. [...] Ces divers étants dont on peut ainsi déterminer l'être-l'un-dans-l'autre ["In"-einandersein] ont tous le même et unique mode d'être de l'être-sous-lamain [Vorhandenseins], en tant que choses survenant à l'intérieur du monde

44. Les problèmes fondamentaux de la phénoménologie, traduction par J.-F. Courtine, Paris, Gallimard, 1985, p. 196-197; Ga. 24, p. 226.

45. Cela signifie que l'intériorité, en 1927, n'est pas l'autre ou le contraire de l'extériorité, du transcendant, mais son envers : le réfléchissement du soi dans le monde est corrélatif de l'ouverture du monde.

46. Etre et temps, p. 248 ; Sein und Zeit, p. 321. 
[“innerhalb” der Welt vorkommende Dinge]. [...] L'être-à... au contraire, désigne une constitution d'être du Dasein, c'est un existential $»^{47}$.

On comprend ici pourquoi Heidegger, contre Husserl, refuse de décrire la propriété du Dasein de se posséder soi-même en terme d' «inclusion» d'un vécu dans un autre : cela reviendrait à comprendre le Dasein comme un Vorhandene, un étant-sous-la-main. Le Dasein, en 1927, est sans intériorité au sens traditionnel du terme, précisément parce qu'il est celui par qui il y a de l'espace, de l'intériorité, des étants intramondains. Il n'est dans aucun lieu. Pour autant, nous l'avons dit, on peut identifier «dans» le Dasein des «résidus» d'intériorité (mis au service d'une nouvelle pensée de l'intériorité). Le deuxième se manifeste dans la seconde forme d'isolement évoquée au paragraphe 64. Pour cette seconde forme, Heidegger n'emploie pas le même terme: il ne parle plus d'un Dasein isoliert, mais de la Vereinzelung du Dasein, qu'on rend également en français par le terme d'isolement $^{48}$. Si le Dasein n'est pas un sujet intérieur isolé du monde, il connaît une autre forme d'isolement dans la résolution devançante, et plus précisément dans l'affection de l'angoisse (dans laquelle s'annonce le soi, le Selbst authentique): "Le Dasein est authentiquement lui-même [ist eigentlich selbst] dans l'isolement originaire [der ursprünglichen Vereinzelung] de cette résolution ré-ticente qui s'intime à elle-même l'angoisse $\gg{ }^{49}$. Dans l'affection originaire de l'angoisse, le Dasein s'éprouve comme isolé des étants intramondains, au sens où leur étrangeté lui devient manifeste. Mais l'important est que, ainsi isolé des autres étants, le Dasein conserve son caractère d'être-au-monde : car précisément l'angoisse surgit devant l'être-au-monde du Dasein. Dans l'instant de l'isolement angoissé, le Dasein est donc doté d'une certaine forme d'intériorité : il est en retrait, séparé des autres étants, mais l'essentiel est que cette séparation n'est pas d'ordre régionale (car ce qui est isolé, c'est l'être-au-monde comme tel).

Il faut analyser, pour terminer, un troisième « résidu » d'intériorité : la totalité (ou la fermeture). En 1927, le Dasein est sans intériorité au sens traditionnel du terme, mais il n'en demeure pas moins une totalité (Ganzheit), c'est-à-dire un être doté, quoiqu'en un sens particulier, de contenus, de parties. Cette totalité se manifeste une nouvelle fois dans l'angoisse: celle-ci amène le Dasein devant lui-même, ouvrant intégralement cet étant. Mais quel sens faut-il alors donner à la Ganzheit du Dasein ? Heidegger apporte une réponse à ces questions dans ses analyses relatives à l'être-pour-la-mort. Nous allons nous concentrer plus précisément sur le paragraphe 48 («Excédent, fin et totalité »[Ausstand, Ende und Ganzheit]).

47. Ibid., p. 62-63; Sein und Zeit, p. 53-54.

48. L'allemand semble cependant insister davantage ici sur l'unicité, sur la solitude du Dasein : einzeln signifie en effet « seul », « séparé », « individuel ».

49. Etre et temps, p. 249 ; Sein und Zeit, p. 322. 
La position du problème de la mort, dans Être et temps, résulte de la nécessité de former un concept de totalité conforme à l'être du Dasein. Or, l'idée de totalité répugne manifestement à l'être-en-avant-de-soi constant du Dasein. Celui-ci ne pourrait pas constituer une totalité, parce que, aussi longtemps qu'il est, il n'a pas atteint sa totalité (il est en-vue-de-lui-même; il y a donc, "dans » le Dasein, des excédents), et lorsqu'il est une totalité (lorsqu'il meurt), il n'est plus, il est devenu un ne-plus-être-là. Heidegger résout cette difficulté en repensant l'être de l'excédent et de la totalité à la lumière de l'être du Dasein. Plus exactement, il faut dire que le Dasein n'a pas d'excédents. Parler d'excédent au sujet du Dasein revient en effet à le considérer comme une " somme » [Summe], c'est-à-dire comme un étant-àportée-de-la-main (Zuhandene). Le Dasein n'est pas une somme, parce qu'il est toujours-déjà ce qu'il n'est pas, sur le mode du pouvoir-être, de l'enavant-de-soi (tandis qu'une somme, selon Heidegger, est " déjà en quelque manière et quelque part à-portée-de-la-main $\gg{ }^{50}$ lorsque certaines de ses parties sont absentes). Mais si le Dasein n'a pas d'excédents, faut-il dire alors, plus largement, qu'il n'a pas de contenus, de parties ? Non : le Dasein, selon Heidegger, a des parties, qu'il est toujours-déjà, sur le mode du pouvoir-être (seul le Dasein qui se comprend de manière inauthentique, c'est-à-dire comme une somme, peut en fin de compte être dit incomplet). Le Dasein, chez Heidegger, a des parties au sens où il est articulé, où il a une certaine structure (on distingue ainsi, par exemple, trois parties du souci). Heidegger semble puiser chez Husserl ${ }^{51}$ (dans la Recherche Logique III, consacrée à la théorie des touts et des parties), un certain sens de la notion de «partie », qu'il réinvestit en le transformant. Husserl distingue les parties indépendantes (celles qui sont indifférentes à la suppression des parties qui lui sont associées) et les parties dépendantes (celles qui sont inséparables d'autres parties) ${ }^{52}$. On peut dire que si le Dasein n'a pas de parties indépendantes, il a en revanche des parties dépendantes. Heidegger réinvestit donc un certain sens du concept husserlien de "partie», mais en le transformant : il lui ôte sa signification régionale (les parties ne sont pas les parties d'un ensemble sous-la-main, ce qui est le cas chez Husserl, parce qu'il pense la distinction Partie/Tout à partir de la représentation) et, ce qui est lié, sa dimension formelle. En effet, chez Husserl, la dépendance réciproque des parties est fondée sur une loi formelle. Le tout est ainsi compris comme une forme logique, c'est-à-dire vide, universelle (les dépendances des parties relativement au tout, et d'une partie vis-à-vis d'une autre, sont comprises dans ce cadre). Heidegger rompt avec cette conception formelle du tout et des parties. Dans les paragraphes consacrés à l'être-pour-la-mort, il explique ainsi que la structure du Dasein, c'est-à-dire sa totalité, est une structure nécessairement unique, en vertu du sens d'être

50. Ibid., p. 196 ; Sein und Zeit, p. 243.

51. Cf. sur ce point C. Ciocan, « Heidegger, la mort et la totalité », Revue philosophique de la France et de l'étranger, 199, (2009), p. 308, qui renvoie à E. Øverenget, Seeing the Self. Heidegger on Subjectivity, Dordrecht, Kluwer Academic Publishers, 1998.

52. Cf. Husserl, Recherche logique III, paragraphe 2. 
particulier du Dasein (l'existence). Après avoir repensé l'être-partiel du Dasein, Heidegger repense ainsi son être-tout. La totalité du Dasein n'est ni une cessation, ni une disparition, ni un accomplissement, ni un achèvement, mais elle-même une possibilité en vue de laquelle le Dasein existe, autant qu'il existe. Cela signifie que la totalité n'advient pas au Dasein, elle ne lui est pas donnée comme un fait qui survient, mais, en tant qu'il est en vue de sa totalité, est pour sa totalité (c'est-à-dire pour-lamort), il est son tout aussi longtemps qu'il est (de manière authentique). La totalité du Dasein est ainsi un pouvoir-être-tout. Celui-ci constitue donc le troisième « résidu » d'intériorité propre au Dasein. On peut l'interpréter par ailleurs comme une fermeture, au sens où la mort constitue une fermeture à l'être. Si le Dasein est essentiellement ouvert (en tant qu'être-au-monde), cette ouverture est corrélée à une fermeture plus originelle, en vue de laquelle le Dasein authentique est (cette fermeture n'étant pas à interpréter comme enfermement dans un certain lieu, dans une région d'étants sous-lamain $)^{53}$.

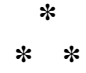

Ainsi, nous avons tenté de montrer comment, à partir des phénomènes de la réflexion et de l'isolement angoissé de l'être-pour-la-mort, Heidegger se réapproprie l'idée traditionnelle d'intériorité : il la repense comme réfléchissement de soi dans le monde, isolement de l'être-au-monde et totalité (ou fermeture) non régionale. Il tente de penser une intériorité de l'être-au-monde. Cette persistance de l'intériorité, dans les cours des années 1920 comme dans l'Hauptwerk de 1927, est l'indice d'une autre persistance : celle du Je, du soi, ou plus exactement du Selbst (littéralement : le même $)^{54}$. Le Dasein n'est absolument pas un sujet, au sens du subjectum ${ }^{55}$,

53. Sur la «fermeture» du Dasein, cf. F. Dastur, Heidegger et la question du temps, Paris, PUF, 1990, p. 59-60.

54. De ce point de vue, on peut dire que si le Dasein échappe à l'histoire du sujet, il s'inscrit pleinement, en revanche, dans celle du je. Sur la distinction de ces deux histoires, cf. notamment l'article d'A. de Libera, "Augustin critique d'Averroès. Deux modèles du sujet au Moyen Âge », in Intellect et Imagination dans la philosophie médiévale, Actes du $X I^{e}$ Congrès international de philosophie médiévale de la SIEPM, Porto, du 26 au 31 août 2002, M.C. Pacheco et J.F. Meirinhos (éds), vol. 1, Turnhout, Brepols (Rencontres de philosophie médiévale, XI, 1), p. 203-246. En tant que je ou soi, le Dasein (qui existe de manière authentique) possède un certain «maintien » (Ständigkeit), une "solidité constante » (beständige Standfestigkeit) : cf. sur ce point Sein und Zeit, p. 322. Dans le même passage, Heidegger affirme même que "[1]es caractères de la "simplicité" [Simplizität], de la "substantialité" [Substantialität], et de la "personnalité" [Personalität], que Kant, par exemple, met à la base de sa doctrine des "Paralogismes de la raison pure" procèdent d'une expérience pré-phénoménologique authentique [einer echten vorphänomenologischen Erfahrung]. La question reste seulement de savoir si ce qui est ainsi ontiquement expérimenté peut être ontologiquement interprété à l'aide des "catégories" citées » (Être et temps, p. 246-247; Sein und Zeit, p. 318).

55. Cf. sur ce point «La métaphysique comme histoire de l'être», in Nietzsche, t. 2, traduction par P. Klossowski, Paris, Gallimard, 1971, p. 319-365, et la distinction entre Subjectität et Subjektivität. 
pour autant, il est un soi $^{56}$. Au paragraphe 64 d'Être et temps (« Souci et ipséité »), Heidegger se demande en quoi peut consister l'unité d'un étant dont l'être a été déterminé comme souci. Il affirme que c'est en tant que le Dasein possède une Selbstheit qu'il est un, et il prend immédiatement soin de distinguer l' « être-soi » de l'« être-sujet».

Pour penser l'intériorité du Dasein, il faut, comme nous avons tenté de le montrer, rompre avec la philosophie scolastique. Quelles sont, dès lors, les sources de cette nouvelle pensée ? Cela pourrait faire l'objet d'un autre travail. Indiquons simplement pour terminer que Heidegger puise notamment ce nouveau sens de l'intériorité dans une certaine pensée religieuse ${ }^{57}$.

56. Le soi, cependant, ne doit pas être compris comme une entité, en quelque sens que ce soit.
À cet égard, la formule das Selbst, employée par Heidegger, peut s'avérer trompeuse.
L'expression Selbstheit semble plus conforme à l'être du soi heideggérien : elle souligne le
fait que le soi n'est pas une certaine entité, un être quelconque, mais une manière d'être.
Cf. sur ce point C. Romano, "Heidegger et Wittgenstein : approches croisées sur la question
de l'ipséité », conférence prononcée dans le cadre du colloque "Heidegger, Wittgenstein,
Mulhall : chemins croisés » organisé à l'Institut Catholique de Paris les 5 et 6 Novembre
2015. 57. Cf. notamment, sur le christianisme originel, Ga. 58, p. 61 : « le paradigme historique le plus profond de ce curieux processus d'installation du centre de gravité de la vie facticielle [faktischen Lebens] et du monde de la vie [Lebenswelt] dans le monde du soi [Selbstwelt] et dans le monde des expériences internes [die Welt der inneren Erfahrungen] s'offre à nous dans la genèse du christianisme. Le monde du soi comme tel entre dans la vie et est vécu comme tel ». 